PART IV

SATELIITES OF JUPITER AND SATURN, AND ARTIFICIAL SATELLITES 


\title{
AN APPLICATION OF THE STROBOSCOPIC METHOD
}

\author{
E. A. Roth \\ ESOC, Darmstadt
}

\section{ABSTRACT}

In this paper the motion of an orbiter of a satellite of one of the major planets is considered. The orbiter undergoes various perturbing effects. It is shown that the semi-analytical stroboscopic method is well suited to take into account all perturbations.

\section{INTRODUCTION}

The stroboscopic method is a semi-analytical method for orbit computation. The denomination "stroboscopic" is derived from the fact that the osculating orbital elements are only known at one well-defined point of the orbit, usually at perigee. The main advantages are the possibility of an easy inclusion of all types of perturbations, the speed of computation and the fair accuracy obtained even after many hundreds of revolutions. In this paper the essential steps for the application of the method will be given.

The example to be considered here is a spacecraft, the orbiter, moving around one of the large satellites (such as JI to JIV, or SVI, or NI) of a major planet (Jupiter, Saturn or Neptun). Such orbiters are technically feasible within the next decade. For various reasons the pericentre will be low and the eccentricity and the inclination can have arbitrary, large values :

$$
\begin{aligned}
& 0<e<e^{*}<1 \\
& 0<i<\pi
\end{aligned}
$$

The upper limit $e^{*}$ of the eccentricity is defined by the sphere of influence of the satellite (Roth, 1975).

2. PERTURBATIONS OF AN ORBITER

\section{1}

V. Szebehely (ed.), Dynamics of Planets and Satellites and Theories of Their Motion, 181-188. All Rights Reserved. Copyright $\odot 1978$ by D. Reidel Publishing Company, Dordrecht, Holland. 
An orbiter of a satellite $S_{n}$ will undergo a number of perturbations which determine the evolution of the orbit and therefore its stability. In the case of a Galilean satellite as central body one has to consider at least the following perturbing forces.

$\begin{array}{lc}\begin{array}{l}\text { Perturbation } \\ \text { Oblateness of the satellite } S_{n}\end{array} & \text { Order of magnitude } \\ \text { Third-body perturbations by the } \\ \text { primary } \\ \begin{array}{l}\text { Fourth-body perturbations : Sun } \\ \quad \text { atellites } S_{m}(m \neq n)\end{array} \\ \begin{array}{c}\varepsilon^{2} \\ \text { Oblateness of the primary }\end{array} \\ \begin{array}{c}\varepsilon^{2} \\ \text { Atmospheric drag }\end{array}\end{array}$

The orders of magnitude of the perturbations give only a rough indication. They depend considerably on the size of the orbit, the satellites and the primary planet.

In the following the perturbations by the oblateness of the primary planet is considered as an example. At the end of the paper a few remarks will be made concerning the other perturbations (section 6).

\section{EXPANSION OF THE PERTURBING FUNCTION}

In a first step the perturbing potential has to be expanded in an appropriate way. The potential due to the oblateness of the primary planet is, as usual, expanded in terms of Legendre polynomials(1).

$$
V^{\prime}=-\sum_{n=2}^{\infty} \frac{c_{n}}{a^{n+1}} P_{n}\left(\frac{z}{d}\right)
$$

For Jupiter only the terms $\mathrm{n}=2,4$ and 6 are known to a fair accuracy (Wong, 1975).

It is now necessary to expand the perturbing acceleration

$$
\underline{B}=\frac{\partial V^{\prime}}{\partial \underline{d}}
$$

in terms of the elements of the planet and the orbiter using the wellknown expansion for $\mathrm{d}^{-1}$ (see fig. 1). The algebra is, however,

(1) The main term ( $\mathrm{n}=0$ ) of the potential of the primary planet gives rise to the third-body perturbation mentioned in section 2 . 


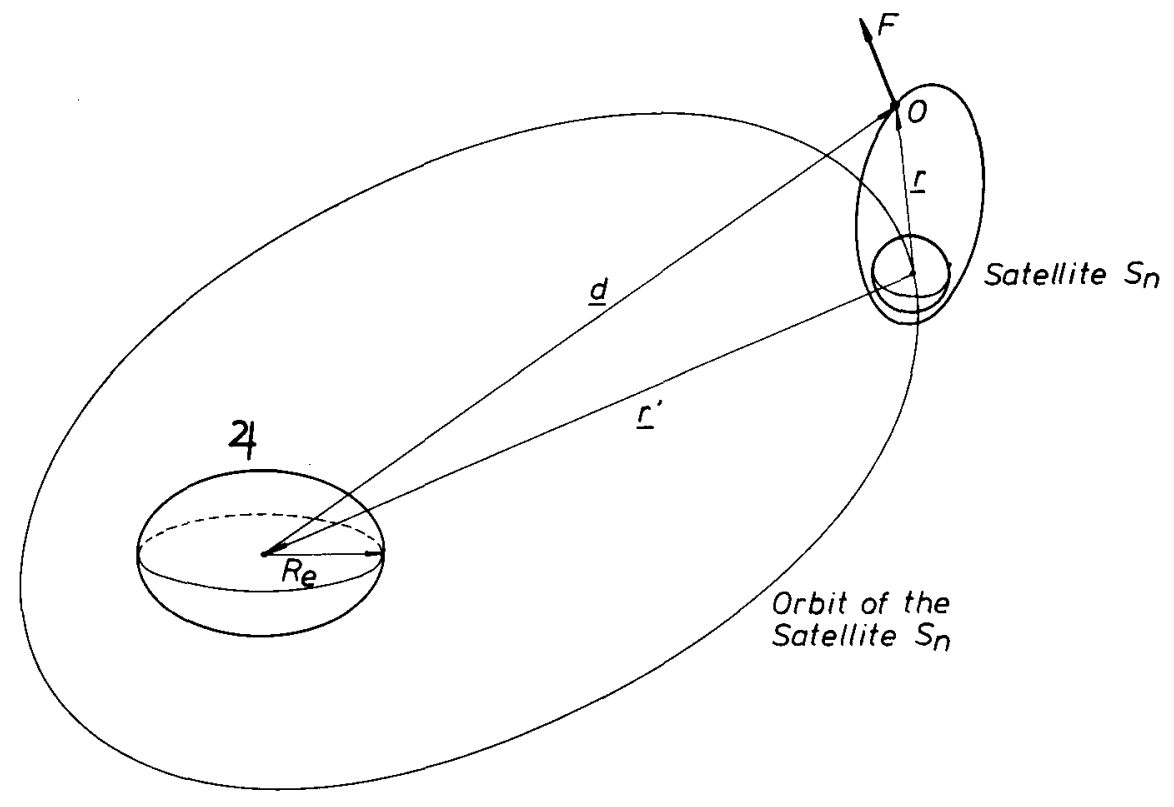

Fig. 1. Orbiter 0 of a satellite $\mathrm{s}_{\mathrm{n}}$.

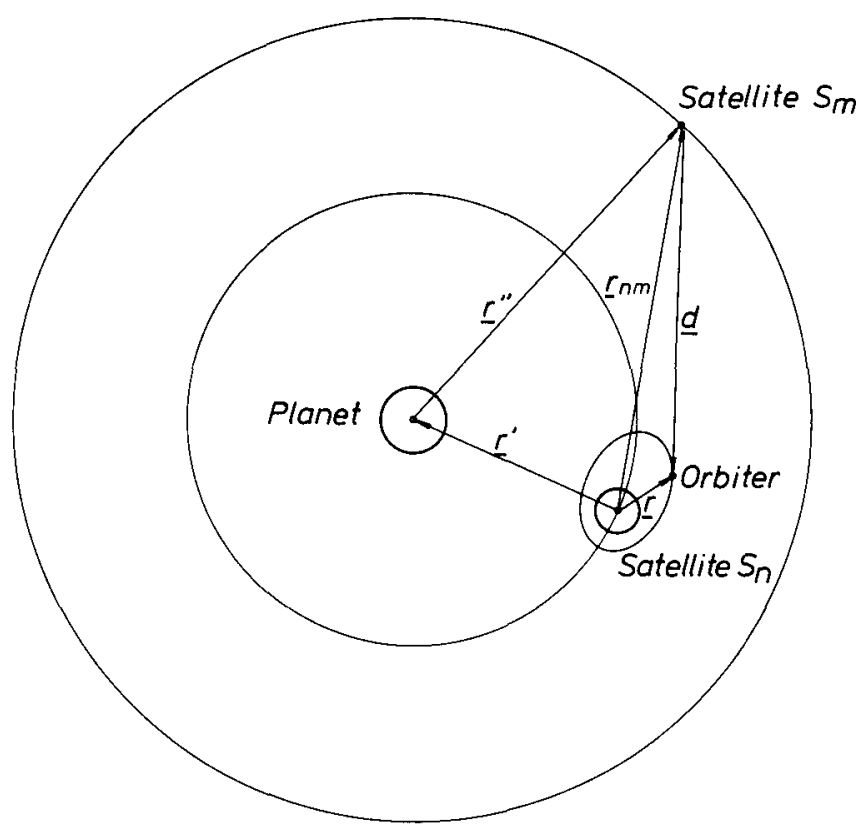

Fig. 2. Geometry of the fourth-body perturbation. 
considerably more involved than in the classical case where the orbiter revolves around the primary planet. It can be shown that the radial, transversal and binormal component of the acceleration due to $J_{n}$ have the form (Roth, 1975)

$$
F_{i}{ }^{(n)}=\frac{k^{(n)}}{p^{\prime n+2}} \sum_{j=0}^{\infty}\left(\frac{p}{p}\right)^{j} \sum_{h} Y_{i j h}^{n}\left(u^{\prime}\right) T_{i j h}(\theta)
$$

where $p=a\left(1-e^{2}\right)$ is the semi-latus rectum of the orbit and $u^{\prime}$ the longitude of the planet (primed quantities refer always to the perturbing primary). $Y_{i, j h}^{n}\left(u^{\prime}\right)$ are polynomials in sin $u^{\prime}$ and $\cos u^{\prime}$, whereas $\mathrm{T}_{i j h}(\theta)$ depends on the true anomaly $\theta$ of the orbiter. The acceleration $F_{i}(n)$ is therefore (in the first approximation) a function of a slow variable $u^{\prime}$ and a fast variable $\theta$. The important step is to consider $Y\left(u^{\prime}\right)$ as a function of the time $t$ and to expand it in a Taylor series. This allows to introduce the mean and the eccentric anomalies $M$ and $E$ of the orbiter. $Y\left(u^{\prime}\right)$ becomes (omitting subscripts for simplicity)

$$
Y=\sum_{s=0}^{\infty} \frac{1}{s !}\left(\frac{T}{2 \pi}\right)^{S} Y^{(s)}\left(t_{0}\right) M^{s}
$$

The derivates $Y^{(S)}$ are evaluated on the osculating orbit of the planet around the satellite (in a system centered at the satellite) and are therefore easily available. Introducing (3.3) into (3.2) it is seen that the perturbing acceleration due to $\mathrm{J}_{\mathrm{n}}$ becomes a double sum over $j$ and $s$

$$
\underline{F}^{n}=\sum_{j=0}^{\infty} \sum_{s=0}^{\infty} \underline{F}_{j s}^{n}
$$

with the obvious abbreviation $F_{j s}^{n}$.

\section{INTEGRATION OF THE LAGRANGEAN EQUATIONS}

The Lagrange equations are used in the Gaussian form and can be written as a matrix equation

$$
\frac{\mathrm{d} E}{\mathrm{~d} \theta}=L \cdot \underline{\mathrm{F}}
$$

It is essential to use orbital elements E, e.g. the Keplerian elements (2) and an angle, such as the true anomaly $\theta$, as independent

(2) If the time is used instead of a time-element, then a second integration becomes necessary. 
variable so that the pericentre is easily defined.

Introducing now the expansion (3.4) into the equation (4.1) it is seen that for each couple $(j, s)$ an equation of the following form is obtained

$$
\frac{d E_{j s}^{n}}{d}=L \cdot F_{j s}^{n}
$$

Considering the expressions (3.2) and (3.3) it is seen that the equations (4.2) have essentially the same form for fixed values $n$ and $j$ and varying $s$. In a first-order perturbation theory the elements are kept constant on the right-hand side of (4.2). It turns out that only integrals of the following type are occuring

$$
I(m, n, q, s ; \theta)=\int_{0}^{\theta} M^{s} \frac{\sin ^{m} \theta \cos ^{n} \theta}{\Delta^{q}} d \theta
$$

with $\Delta=1+e \cos \theta$ and $m+n<q$. Numerous recursion formulae can be derived for these integrals (Sridharan, 1973). It is also possible to introduce the eccentric anomaly $E$ as new independent variable. The integrand of (4.3) becomes in this case a finite Poisson polynomial in $E$ which is easy to integrate. Again recursive relations exist which can be used for the evaluation of the integrals (Roth, 1971, 1974). Obviously, these relations are ideally suited for the application of formula manipulation techniques.

\section{THE STROBOSCOPIC METHOD}

For the application of the stroboscopic method the integrals (4.3) are evaluated between the limits $\mathrm{E}=0$ and $\mathrm{E}=2 \pi$. The integration is therefore performed over one revolution from pericentre to pericentre. This has the advantage to lead to a considerable simplification of the final formulae since many of the terms vanish after integration.

Carrying out the integration provides the variation $\Delta \underline{E}$ of the elements after one complete revolution

$$
\Delta \underline{E}^{n}=\sum_{j} \sum_{s} \Delta \underline{E}_{j s}^{n}
$$

Now, the elements (at pericentre) are updated according to

$$
\underline{E}+\Delta \underline{E}^{n} \Rightarrow \underline{E}
$$

which provides the new initial values. With these new elements and the new time $t+\Delta t^{n}$ it is possible to calculate new values for the 
derivates $Y^{(s)}$ and all other quantities. Performing again the integration the increments $\Delta E$ of the next revolution are obtained. This is repeated until the desired final time is reached.

\section{REMARKS}

1. It has some advantage to keep the indefinite integrals (4.3) since they are needed if the short-period terms have to be recovered. Moreover, they are necessary for the derivation of a second-order perturbation theory.

2. The recursive relations mentioned in section 4 could be used to calculate directly the numerical values of the definite integrals (4.3) with the upper limit $2 \pi$.

3. Updating the elements after each revolution (formula 5.2) implies that the results are somewhat better than with a pure first-order perturbation theory. In addition the contributions of the long-period terms are obtained implicitly.

Obviously the method sketched for the oblateness perturbation $J_{n}$ by the primary planet can be applied in a similar way to the other perturbations $\underline{F}^{\mathrm{q}}$ mentioned in section 2. At each step (each revolution)

all corresponding increments $\Delta \underline{E}^{q}$ are separately calculated and combined to the total increment

$$
\Delta \underline{E}=\sum_{q} \Delta \underline{E}^{q}
$$

It will be necessary to expand the various perturbing forces in the way explained in section 3. However, the expansion (3.3) is only needed if the perturbation is non-conservative. In order to be consistent it is necessary to derive the perturbations to the appropriate order. In the case of forces of the order $\varepsilon$ it will be necessary to use a second-order perturbation theory, at least for the main term $(j=0)$ in an expansion like $(3.2)$.

\section{SURVEY OF THE VARIOUS PERTURBATIONS}

In the following we present shortly the status concerning the various perturbations mentioned in section 2 .

Oblateness of the Satelite $\mathrm{S}$

The increments $\Delta E$ of the first order are given by the wellknown secular terms. In some cases it will be necessary to consider also the 
second-order term $\mathrm{J}_{2}^{2}$ for which convenient expressions have been given by Merson (1963).

Third-body perturbation

The first-order perturbation theory of a third body has been developed by Lidoy (1962) and later extended by Roth (1968, 1971). A secondorder perturbation theory for the main term has however not yet been developed.

Fourth-body perturbation

The geometry of the problem is shown in figure 2. The perturbing body (the sun, the sateliites $S_{m}(m \neq n)$ is moving around the third body

(Jupiter). The distance d depends in this case on the motion of Jupiter, the satellite $S_{m}$ and the orbiter. The expansion of the perturbing for-

ce in the satellite-centered system is considerably more involved as in the third-body case. This expansion is presently under investigation.

Oblateness of the primary planet

The development of the corresponding perturbing function and the derivation of the first-order increments for $J_{2}$ and $J_{4}$ has been given by

$\operatorname{Roth}(1975)$.

Atmospheric drag

The largest satellites of the major planets are known to have an atmosphere. It is therefore necessary to consider the perturbation by the drag. The orbits under consideration will have a relatively low pericentre and a large eccentricity. In this case the developments given by King-Hele $(1962,1964)$ are very satisfactory (see also Roth, 1970).

\section{REFERENCES}

King-Hele, D. G. :

-1962 , The Contraction of Satellite Orbits under the Influence of Air Drag. III. High-eccentricity Orbits $\left(0.2 \leq e^{<1)}\right.$. Proc. Roy. Soc., A, 267, $541-557$

-1964, Theory of Satellite Orbits in an Atmosphere. Butterworth, London

Lidov, M. L. :

-1962, The Evolution of Artificial Satellites of Planets under the 
Action of Gravitational Perturbations of External Bodies, Planet. Space Science, 2, 719-759

Merson, R. H. :

-1963, The Perturbation of a Satellite Orbit in an axi-symmetric gravitational Field. RAE, TN Space 26 ,

Roth, E. A. :

-1968, Luni-solar Perturbations of a Highly Eccentric Orbit Satellite, ESRO SR-9, Paris

-1970, A Method and a Computer Program for the Quick Determination of a Preliminary Launch-Window for Satellites with Moderately-to-Highly Eccentric Orbits, ESRO SR-15, Paris

-1971, The Short Periodic Luni-solar Perturbation of the Motion of a Highly Eccentric Orbit Satellite, ESRO SR-18, Paris

-1973, Fast Computation of High Eccentricity Orbits by the Stroboscopic Method, Cel. Mech. , $8,245-249$

-1974, Perturbation of a Planetary Orbiter by Radiation Pressure, ESRO $\mathrm{SR}-24$, Paris

-1975, Perturbation of a Satellite Orbiter by the Oblateness of the Primary Planet, ESA SR-26, Paris

Sridharan, R. :

-1973, Evolution of the Orbital Elements in Geocentric Orbits of High Eccentricity by Non-Numeric Computation, Ph. D. Thesis, CarnegieMellon University

Wong, S. K. :

-1975, Gravity Field of Jupiter from Pioneer 11 Tracking Data, Science, $188,476-477$ 